\title{
Passerini Multicomponent Reaction of Indane-1,2,3-Trione: an Efficient Route for the One-Pot Synthesis of Sterically Congested 2,2-Disubstituted Indane-1,3-Dione Derivatives
}

\author{
Ali Reza Kazemizadeh and Ali Ramazani*
}

\author{
Chemistry Department, Zanjan University, P.O. Box 45195-313, Zanjan, Iran
}

\begin{abstract}
A reação de Passerini de indano-1,2,3-triona, isocianetos e derivados do ácido benzóico ocorre a temperatura ambiente, conduzindo a derivados estericamente congestionados de indano-1,3-diona 2,2-dissubstituídas em excelentes rendimentos. A reação é limpa, ocorre sob condições brandas e reações laterais não foram observadas.
\end{abstract}

The Passerini reaction of indane-1,2,3-trione, isocyanides and benzoic acid derivatives proceed at room temperature and sterically congested 2,2-disubstituted indane-1,3-dione derivatives are synthesized in excellent yields. The reaction proceeds smoothly and cleanly under mild conditions and no side reactions are observed.

Keywords: indane-1,2,3-trione, isocyanide, Passerini reaction, benzoic acid

\section{Introduction}

In recent years, multicomponent reactions (MCRs) have become important tools in modern preparative synthetic chemistry because these reactions increase the efficiency by combining several operational steps without isolation of intermediates or changes of the conditions. ${ }^{1-6}$ This principle, therefore, is highly efficient in terms of time as well as resources. ${ }^{7}$ Among the known multicomponent reactions to date, the most valuable reactions are those based on isocyanides. Isocyanide-based multicomponent reactions (abbreviated to IMCRs by Ugi and Dömling) by virtue of their synthetic potential, their inherent atom efficiency, convergent nature, ease of implementation, and the generation of molecular diversity, have attracted much attention because of the advantages that they offer to the field of combinatorial chemistry. ${ }^{8}$ IMCRs are particularly interesting because they are more versatile and diverse than the remaining MCRs. The great potential of isocyanides for the development of multicomponent reactions lies in the diversity of available bond forming processes, their functional group tolerance, and the high levels of chemo-, regio-, and stereoselectivity often observed. The outstanding position of IMCRs can be traced back to the exceptional reactivity of the functional group of the isocyanide. No other functional group reacts

*e-mail: aliramazani@yahoo.com with nucleophiles and electrophiles at the same atom, leading to the so-called $\alpha$-adduct. Today most IMCR chemistry relates to the classical reactions of Passerini and Ugi. Indeed, the large number of different scaffolds now available mostly builds on these two IMCRs and their combination with other types of reactions. ${ }^{9-18}$ Passerini reactions involve an oxo component, an isocyanide, and a nucleophile. ${ }^{19-21}$ The Passerini reactions are beginning to find utility in the drug discovery process, and total syntheses of biologically relevant natural products. This reaction has been widely used in synthetic and medicinal chemistry. ${ }^{12}$ For example, it has often been involved as a key step in the total synthesis of natural products due to the fact that the $\alpha$-acyloxycarboxamide group is a frequently recurring motif in many pharmacologically interesting natural products. ${ }^{22}$ The mechanism of the Passerini reaction has been investigated by Baker and Stanonis. ${ }^{23}$ In connection with our recent interest to isocyanide chemistry, ${ }^{24-27}$ we report the Passerini multicomponent reaction between, indane-1,2,3-trione, isocyanides and benzoic acid derivatives in this article.

\section{Results and Discussion}

The indane-1,2,3-trione (1), isocyanides (2) and benzoic acid derivatives (3) in dichloromethane react together in a 1:1:1 ratio at room temperature to produce $\alpha$-acyloxycarboxamides (4a-j) (Scheme 1 and Table 1$)$. 
The reaction proceeds smoothly and cleanly under mild conditions and no side reactions are observed. The pure products $(4 \mathrm{a}-\mathrm{j})$ are stable at room temperature for several months. The structures of the products were deduced from their IR, ${ }^{1} \mathrm{H}$ NMR, ${ }^{13} \mathrm{C}$ NMR and elemental analyses. For example the ${ }^{1} \mathrm{H}$ NMR spectrum of $\mathbf{4 a}$ exhibited distinct signals arising from cyclohexyl $\left(\delta_{\mathrm{H}}=1.19-2.17 \mathrm{ppm}\right)$, $\mathrm{NCH}$ (3.77 ppm), NH (6.58 ppm) and aromatic $\mathrm{CH}$ (7.268.07). The ${ }^{13} \mathrm{C}$ NMR spectrum of $\mathbf{4 a}$ showed 15 distinct resonances arising from $\mathrm{CH}_{2}$ of cyclohexyl $(24.64,25.34$, $32.66 \mathrm{ppm}), \mathrm{NCH}$ (49.03 ppm), C-O (84.08 ppm), aromatic carbons $(124.08,127.01,128.83,130.15,134.51,136.07$ and $141.50 \mathrm{ppm}), \mathrm{CO}$ of ester (161.53 ppm), CO of amide (163.84 ppm), CO of ketone (191.60 ppm). The IR spectrum showed an $\mathrm{NH}$ absorption at $3331 \mathrm{~cm}^{-1}$. The mass spectrum of $\mathbf{4 a}$ displayed a molecular ion peak at $m / z: 391$ value.

\section{Experimental}

Starting materials and solvents were obtained from Merck (Germany) and Fluka (Switzerland) and were used without further purification. Melting points were measured on an Electrothermal 9100 apparatus and are uncorrected. IR spectra were measured on a Shimadzu IR-460 spectrometer. ${ }^{1} \mathrm{H}$ and ${ }^{13} \mathrm{C}$ NMR spectra were measured $\left(\mathrm{CDCl}_{3}\right.$ solution)

Table 1. Synthesis of $\alpha$-acyloxycarboxamides (4a-j) (see Scheme 1)

\begin{tabular}{lccccc}
\hline Entry & Products & $\mathrm{R}$ & $\mathrm{X}$ & $\mathrm{Y}$ & Yield / (\%) \\
\hline 1 & $\mathbf{4 a}$ & cyclohexyl & $\mathrm{H}$ & $\mathrm{H}$ & 97 \\
2 & $\mathbf{4 b}$ & cyclohexyl & $\mathrm{H}$ & $\mathrm{Me}$ & 95 \\
3 & $\mathbf{4 c}$ & cyclohexyl & $\mathrm{Me}$ & $\mathrm{H}$ & 96 \\
4 & $\mathbf{4 d}$ & cyclohexyl & $\mathrm{H}$ & $\mathrm{Br}$ & 96 \\
5 & $\mathbf{4 e}$ & cyclohexyl & $\mathrm{Br}$ & $\mathrm{H}$ & 95 \\
6 & $\mathbf{4 f}$ & $t$-Bu & $\mathrm{H}$ & $\mathrm{H}$ & 97 \\
7 & $\mathbf{4 g}$ & $t$-Bu & $\mathrm{H}$ & $\mathrm{Me}$ & 96 \\
8 & $\mathbf{4 h}$ & $t$-Bu & $\mathrm{Me}$ & $\mathrm{H}$ & 97 \\
8 & $\mathbf{4 i}$ & $t$-Bu & $\mathrm{H}$ & $\mathrm{Br}$ & 95 \\
10 & $\mathbf{4 j}$ & $t$-Bu & $\mathrm{Br}$ & $\mathrm{H}$ & 95 \\
\hline
\end{tabular}

with a BRUKER DRX-250 AVANCE spectrometer at 250.0 and $62.5 \mathrm{MHz}$, respectively. Mass spectra were recorded on a FINNIGAN-MAT 8430 mass spectrometer operating at an ionization potential of $20 \mathrm{eV}$. Elemental analyses were performed using a Heraeus CHN-O-Rapid analyzer. The results agreed favorably with the calculated values.

\section{General procedure}

To a magnetically stirred solution of indane-1,2,3-trione (1) $(0.2 \mathrm{mmol})$ and benzoic acid derivatives $(\mathbf{3})(0.2 \mathrm{mmol})$ in dry $\mathrm{CH}_{2} \mathrm{Cl}_{2}(5 \mathrm{~mL})$ was added dropwise a solution of isocyanides (2) $(0.2 \mathrm{mmol})$ in $\mathrm{CH}_{2} \mathrm{Cl}_{2}(2 \mathrm{~mL})$ at room temperature over $10 \mathrm{~min}$. The mixture was stirred for 1 to 2 hours at room temperature. The solvent was removed under reduced pressure and pure products $(\mathbf{4 a} \mathbf{a}-\mathbf{j})$ were obtained. The characterization data of the compounds are given below.

2-[(Cyclohexylamino) carbonyl]-1,3-dioxo-2,3-dihydro$1 H$-indene-2-yl benzoate (4a)

Yield 97\%; white powder, mp 167.9-170.0 ${ }^{\circ} \mathrm{C}$; IR ( $\left.\mathrm{KBr}\right)$ $v_{\max } / \mathrm{cm}^{-1}: 3331(\mathrm{NH}), 2931,2854,1731,1654,1531,1277$; ${ }^{1} \mathrm{H}$ NMR $\left(250 \mathrm{MHz}, \mathrm{CDCl}_{3}\right) \delta 1.19-2.17\left(\mathrm{~m}, 10 \mathrm{H}, 5 \mathrm{CH}_{2}\right.$ of cyclohexyl), 3.77 (m, 1 H, N-CH), $6.58(\mathrm{~d}, J 7.0 \mathrm{~Hz}$, $1 \mathrm{H}, \mathrm{NH}), 7.26-8.07$ (m, $9 \mathrm{H}$, arom $\mathrm{CH}) .{ }^{13} \mathrm{C}$ NMR $(62.5$ $\left.\mathrm{MHz}, \mathrm{CDCl}_{3}\right) \delta 24.64,25.34,32.66\left(\mathrm{CH}_{2}\right.$ of cyclohexyl), $49.03(\mathrm{NCH}), 84.08(\mathrm{C}-\mathrm{O}), 124.08,127.01,128.83,130.15$, $134.51,136.07$ and 141.50 (aromatic carbons), 161.53 (CO of ester), 163.84 (CO of amide), 191.60 (CO of ketone; MS (EI, $20 \mathrm{eV}): m / z(\%)=391(2)\left(\mathrm{M}^{+}\right), 269(1), 161$ (3), 133 (2), 105 (100), 77 (22), 55 (10). Found: C, 70.65; H, 5.36; N, 3.51. Calc. for $\mathrm{C}_{23} \mathrm{H}_{21} \mathrm{NO}_{5}: \mathrm{C}, 70.58 ; \mathrm{H}, 5.41 ; \mathrm{N}$, $3.58 \%$.

2-[(Cyclohexylamino $)$ carbonyl]-1,3-dioxo-2,3-dihydro1H-indene-2-yl 4-methylbenzoate (4b)

Yield 95\%; white powder, mp 220.8-222.0 ${ }^{\circ} \mathrm{C}(\mathrm{dec})$; IR (KBr) $v_{\max } / \mathrm{cm}^{-1}: 3338(\mathrm{NH}), 2938,2854,1731,1646$, 1523,$1277 ;{ }^{1} \mathrm{H}$ NMR $\left(250 \mathrm{MHz}, \mathrm{CDCl}_{3}\right) \delta 1.23-1.98$ (m,<smiles>[R]NC(=O)C1(OC(=O)c2ccc([Y])c([X])c2)C(=O)c2ccccc2C1=O</smiles>

(1)

(2)

(3)

$(4 \mathbf{a}-\mathbf{j})$

Scheme 1. Passerini multicomponent reaction of indane-1,2,3-trione (see Table 1). 
$10 \mathrm{H}, 5 \mathrm{CH}_{2}$ of cyclohexyl), $2.44\left(\mathrm{~s}, 3 \mathrm{H}, \mathrm{CH}_{3}\right), 3.75(\mathrm{~m}$, $1 \mathrm{H}, \mathrm{N}-\mathrm{CH}), 6.56(\mathrm{~d}, J 8.0 \mathrm{~Hz}, 1 \mathrm{H}, \mathrm{NH}), 7.27-8.08$ (m, $8 \mathrm{H}$, arom $\mathrm{CH}) .{ }^{13} \mathrm{C}$ NMR $\left(62.5 \mathrm{MHz}, \mathrm{CDCl}_{3}\right) \delta 21.89$ $\left(\mathrm{CH}_{3}\right), 24.64,25.35,32.66\left(\mathrm{CH}_{2}\right.$ of cyclohexyl $), 48.98$ $(\mathrm{NCH}), 83.92(\mathrm{C}-\mathrm{O}), 124.07,124.15,129.54,130.21$, $136.03,141.50$ and 145.63 (aromatic carbons), 161.61 (CO of ester), 163.88 (CO of amide), 191.75 (CO of ketone). Found: C, 71.04; $\mathrm{H}, 5.76 ; \mathrm{N}, 3.38$. Calc. for $\mathrm{C}_{24} \mathrm{H}_{23} \mathrm{NO}_{5}$ : C, $71.10 ; \mathrm{H}, 5.72 ; \mathrm{N}, 3.45 \%$.

2-[(Cyclohexylamino $)$ carbonyl $]-1,3-$ dioxo-2,3-dihydro$1 H$-indene-2-yl3-methylbenzoate $(\mathbf{4 c})$

Yield 96\%; white powder, mp 175.0-177. $1^{\circ} \mathrm{C}$; IR (KBr) $v_{\max } / \mathrm{cm}^{-1}: 3307(\mathrm{NH}), 2931,2854,1731,1654,1538,1285$; ${ }^{1} \mathrm{H}$ NMR $\left(250 \mathrm{MHz}, \mathrm{CDCl}_{3}\right) \delta 1.25-1.98\left(\mathrm{~m}, 10 \mathrm{H}, 5 \mathrm{CH}_{2}\right.$ of cyclohexyl), $2.40\left(\mathrm{~s}, 3 \mathrm{H}, \mathrm{CH}_{3}\right), 3.77(\mathrm{~m}, 1 \mathrm{H}, \mathrm{N}-\mathrm{CH})$, $6.58(\mathrm{~d}, J 7.5 \mathrm{~Hz}, 1 \mathrm{H}, \mathrm{NH}), 7.26-8.08(\mathrm{~m}, 8 \mathrm{H}$, arom $\mathrm{CH})$. ${ }^{13} \mathrm{C} \mathrm{NMR}\left(62.5 \mathrm{MHz}, \mathrm{CDCl}_{3}\right) \delta 21.33\left(\mathrm{CH}_{3}\right), 24.60,25.35$, $32.61\left(\mathrm{CH}_{2}\right.$ of cyclohexyl), $48.95(\mathrm{NCH}), 84.01(\mathrm{C}-\mathrm{O})$, 124.07, 126.87, 127.26, 128.71, 130.77, 135.32, 136.06, $138.71,141.50$ (aromatic carbons), 161.56 (CO of ester), 164.00 (CO of amide), 191.67 (CO of ketone). Found: C, 71.13; H, 5.77; N, 3.36. Calc. for $\mathrm{C}_{24} \mathrm{H}_{23} \mathrm{NO}_{5}: \mathrm{C}, 71.10 ; \mathrm{H}$, $5.72 ; \mathrm{N}, 3.45 \%$.

2-[(Cyclohexylamino)carbonyl]-1,3-dioxo-2,3-dihydro1H-indene-2-yl 4-bromobenzoate (4d)

Yield 96\%; white powder, mp 211.1-212.7 ${ }^{\circ} \mathrm{C}$ (dec); IR (KBr) $v_{\text {max }} / \mathrm{cm}^{-1}: 3331(\mathrm{NH}), 2938,2854,1731,1646$, 1592, 1277; ${ }^{1} \mathrm{H}$ NMR (250 MHz, $\left.\mathrm{CDCl}_{3}\right) \delta 1.18-1.97$ (m, $10 \mathrm{H}, 5 \mathrm{CH}_{2}$ of cyclohexyl), $3.76(\mathrm{~m}, 1 \mathrm{H}, \mathrm{N}-\mathrm{CH}), 6.50$ (d, J 8.0 Hz, $1 \mathrm{H}, \mathrm{NH}), 7.26-8.08(\mathrm{~m}, 8 \mathrm{H}$, arom $\mathrm{CH}) .{ }^{13} \mathrm{C}$ NMR $\left(62.5 \mathrm{MHz}, \mathrm{CDCl}_{3}\right) \delta 24.66,25.31,32.66\left(\mathrm{CH}_{2}\right.$ of cyclohexyl), 49.09 ( $\mathrm{NCH}), 84.24$ (C-O), 124.13, 125.88, $129.95,131.53,132.27,136.20,141.43$, (aromatic carbons), 161.29(CO of ester), 163.24 (CO of amide), 191.39 (CO of ketone). Found: C, 58.80; H, 4.32; Br, 16.95; N, 2.95. Calc. for $\mathrm{C}_{23} \mathrm{H}_{20} \mathrm{BrNO}_{5}$ : C, 58.74; $\mathrm{H}, 4.29 ; \mathrm{Br}, 16.99 ; \mathrm{N}, 2.98 \%$.

2-[(Cyclohexylamino) carbonyl]-1,3-dioxo-2,3-dihydro1H-indene-2-yl 3-bromobenzoate (4e)

Yield 95\%; white powder, mp 188.6-190.0 ${ }^{\circ} \mathrm{C}(\mathrm{dec})$; IR (KBr) $v_{\text {max }} / \mathrm{cm}^{-1}: 3385(\mathrm{NH}), 2931,2854,1731,1669$, 1531, 1262; ${ }^{1} \mathrm{H}$ NMR (250 MHz, $\left.\mathrm{CDCl}_{3}\right) \delta 1.25-1.97$ (m, $10 \mathrm{H}, 5 \mathrm{CH}_{2}$ of cyclohexyl), $3.77(\mathrm{~m}, 1 \mathrm{H}, \mathrm{N}-\mathrm{CH}), 6.51$ (d, J 7.0 Hz, $1 \mathrm{H}, \mathrm{NH}), 7.26-8.12(\mathrm{~m}, 9 \mathrm{H}$, arom $\mathrm{CH}) .{ }^{13} \mathrm{C}$ NMR $\left(62.5 \mathrm{MHz}, \mathrm{CDCl}_{3}\right) \delta 24.59,25.32,32.59\left(\mathrm{CH}_{2}\right.$ of cyclohexyl), $49.06(\mathrm{NCH}), 84.31$ (C-O), 122.83, 124.15, 128.66, 128.90, 130.40, 133.16, 136.21, 137.44, 141.44 (aromatic carbons), 161.22(CO of ester), 162.58 (CO of amide), 191.26 (CO of ketone). Found: C, 58.72; H, 4.33;
$\mathrm{Br}, 17.05 ; \mathrm{N}, 2.94$. Calc. for $\mathrm{C}_{23} \mathrm{H}_{20} \mathrm{BrNO}_{5}: \mathrm{C}, 58.74 ; \mathrm{H}$, 4.29; Br, 16.99; N, $2.98 \%$.

2-[(tert-Butylamino)carbonyl]-1,3-dioxo-2,3-dihydro- $1 \mathrm{H}$ indene-2-yl benzoate (4f)

Yield 97\%; white powder, mp 186.0-187. ${ }^{\circ} \mathrm{C}(\mathrm{dec})$; IR (KBr) $v_{\max } / \mathrm{cm}^{-1}: 3408(\mathrm{NH}), 2969,1723,1685,1531$, 1292; ' ${ }^{\mathrm{H}} \mathrm{NMR}\left(250 \mathrm{MHz}, \mathrm{CDCl}_{3}\right) \delta 1.42(\mathrm{~s}, 9 \mathrm{H}, t$-Bu); $6.55(\mathrm{~s}, 1 \mathrm{H}, \mathrm{NH}) ; 7.26-8.06(\mathrm{~m}, 9 \mathrm{H}$, arom $\mathrm{CH}) .{ }^{13} \mathrm{C} \mathrm{NMR}$ $\left(62.5 \mathrm{MHz}, \mathrm{CDCl}_{3}\right) \delta 28.56\left(\mathrm{CMe}_{3}\right) ; 52.67(\mathrm{~N}-\mathrm{C}) ; 84.09$ (C-O); 124.06, 127.00, 128.84, 130.11, 134.52, 136.05 and 141.66 (aromatic carbons); 161.63 (CO of ester); 163.73 (CO of amide); 191.84 (CO of ketone). Found: C, 68.96; $\mathrm{H}, 5.26 ; \mathrm{N}, 3.89$. Calc. for $\mathrm{C}_{21} \mathrm{H}_{19} \mathrm{NO}_{5}: \mathrm{C}, 69.03 ; \mathrm{H}, 5.24$; $\mathrm{N}, 3.83 \%$.

2-[(tert-Butylamino)carbonyl]-1,3-dioxo-2,3-dihydro- $1 \mathrm{H}$ indene-2-yl 4-methylbenzoate (4g)

Yield 96\%; white powder, $\mathrm{mp} 50.5-152.0{ }^{\circ} \mathrm{C}$ (dec); IR $(\mathrm{KBr}) v_{\text {max }} / \mathrm{cm}^{-1}: 3346(\mathrm{NH}), 2977,1731,1677,1523,1292$; ${ }^{1} \mathrm{H} \mathrm{NMR}\left(250 \mathrm{MHz}, \mathrm{CDCl}_{3}\right) \delta 1.41$ (s, $\left.9 \mathrm{H}, t-\mathrm{Bu}\right) ; 2.44$ (s, $\left.3 \mathrm{H}, \mathrm{CH}_{3}\right) ; 6.53(\mathrm{~s}, 1 \mathrm{H}, \mathrm{NH}) ; 7.26-8.08(\mathrm{~m}, 8 \mathrm{H}$, arom $\mathrm{CH})$. ${ }^{13} \mathrm{CNMR}\left(62.5 \mathrm{MHz}, \mathrm{CDCl}_{3}\right) \delta 21.84\left(\mathrm{CH}_{3}\right) ; 28.56\left(\mathrm{CMe}_{3}\right)$; $52.60(\mathrm{~N}-\mathrm{C}) ; 83.94$ (C-O); 124.01, 124.20, 129.54, 130.15, $135.95,141.68$ and 145.59 (aromatic carbons); 161.73 (CO of ester); 163.75 (CO of amide); 191.94 (CO of ketone). Found: C, 69.70; $\mathrm{H}, 5.55 ; \mathrm{N}, 3.72$. Calc. for $\mathrm{C}_{22} \mathrm{H}_{21} \mathrm{NO}_{5}$ : $\mathrm{C}$, 69.65; H, 5.58; N, 3.69\%.

2-[(tert-Butylamino)carbonyl]-1,3-dioxo-2,3-dihydro- $1 \mathrm{H}$ indene-2-yl 3-methylbenzoate (4h)

Yield 97\%; white powder, $\mathrm{mp} 169.3-170.7^{\circ} \mathrm{C}(\mathrm{dec})$; IR $(\mathrm{KBr}) v_{\max } / \mathrm{cm}^{-1}: 3431(\mathrm{NH}), 2969,1731,1685,1523,1292$; ${ }^{1} \mathrm{H}$ NMR $\left(250 \mathrm{MHz}, \mathrm{CDCl}_{3}\right) \delta 1.42(\mathrm{~s}, 9 \mathrm{H}, t-\mathrm{Bu}) ; 2.41$ (s, $\left.3 \mathrm{H}, \mathrm{CH}_{3}\right) ; 6.55(\mathrm{~s}, 1 \mathrm{H}, \mathrm{NH}) ; 7.26-8.08(\mathrm{~m}, 8 \mathrm{H}$, arom $\mathrm{CH})$. ${ }^{13} \mathrm{CNMR}\left(62.5 \mathrm{MHz}, \mathrm{CDCl}_{3}\right) \delta 21.30\left(\mathrm{CH}_{3}\right) ; 28.55\left(\mathrm{CMe}_{3}\right)$; $52.62(\mathrm{~N}-\mathrm{C}) ; 84.01$ (C-O); 124.02, 126.90, 127.20, 128.70 , 130.73, 135.28, 135.99, 138.70 and 141.68 (aromatic carbons); 161.67 (CO of ester); 163.86 (CO of amide); 191.87 (CO of ketone). Found: C, 69.71; H, 5.60; N, 3.65. Calc. for $\mathrm{C}_{22} \mathrm{H}_{21} \mathrm{NO}_{5}: \mathrm{C}, 69.65 ; \mathrm{H}, 5.58 ; \mathrm{N}, 3.69 \%$.

2-[(tert-Butylamino)carbonyl]-1,3-dioxo-2,3-dihydro- $1 \mathrm{H}$ indene-2-yl 4-bromobenzoate (4i)

Yield 95\%; white powder, $\mathrm{mp} 213.0-214.7^{\circ} \mathrm{C}$ (dec); IR $(\mathrm{KBr}) v_{\max } / \mathrm{cm}^{-1}: 3431(\mathrm{NH}), 2977,1731,1685,1592,1523$, 1277; ' ${ }^{\mathrm{H}} \mathrm{NMR}\left(250 \mathrm{MHz}, \mathrm{CDCl}_{3}\right) \delta 1.41$ (s, $9 \mathrm{H}, t$-Bu); 6.45 (s, $1 \mathrm{H}, \mathrm{NH}) ; 7.26-8.08(\mathrm{~m}, 8 \mathrm{H}$, arom $\mathrm{CH}) .{ }^{13} \mathrm{C} \mathrm{NMR}$ $\left(62.5 \mathrm{MHz}, \mathrm{CDCl}_{3}\right) \delta 28.55\left(\mathrm{CMe}_{3}\right) ; 52.73(\mathrm{~N}-\mathrm{C}) ; 84.25$ (C-O); 124.08, 125.92, 129.93, 131.46, 132.28, 136.12 and 141.60 (aromatic carbons); 161.40 (CO of ester); 163.11 
(CO of amide); 191.58 (CO of ketone). Found: $\mathrm{C}, 56.73 ; \mathrm{H}$, 4.10; $\mathrm{Br}, 17.96 ; \mathrm{N}, 3.09$. Calc. for $\mathrm{C}_{21} \mathrm{H}_{18} \mathrm{BrNO}_{5}$ : C, 56.77; H, 4.08; Br, 17.99; N, 3.15\%.

2-[(tert-Butylamino)carbonyl]-1,3-dioxo-2,3-dihydro-1Hindene-2-yl 3-bromobenzoate (4j)

Yield 95\%; white powder, mp 184.8-185.8 ${ }^{\circ} \mathrm{C}(\mathrm{dec})$; IR (KBr) $v_{\text {max }} / \mathrm{cm}^{-1}: 3423(\mathrm{NH}), 2969,1731,1677,1531$, 1262; ${ }^{1} \mathrm{H}$ NMR (250 MHz, $\left.\mathrm{CDCl}_{3}\right) \delta 1.42(\mathrm{~s}, 9 \mathrm{H}, t-\mathrm{Bu})$; 6.46 (s, $1 \mathrm{H}, \mathrm{NH}) ; 7.26-8.12(\mathrm{~m}, 8 \mathrm{H}$, arom $\mathrm{CH}) .{ }^{13} \mathrm{C} \mathrm{NMR}$ $\left(62.5 \mathrm{MHz}, \mathrm{CDCl}_{3}\right) \delta 28.54\left(\mathrm{CMe}_{3}\right) ; 52.77(\mathrm{~N}-\mathrm{C}) ; 84.31$ (C-O); 122.84, 124.10, 128.59, 128.93, 130.39, 133.15, $136.15,137.42$ and 141.62 (aromatic carbons); 161.32 (CO of ester); 162.44 (CO of amide); 191.46 (CO of ketone). Found: C, 56.83; H, 4.10; Br, 18.02; N, 3.10. Calc. for $\mathrm{C}_{21} \mathrm{H}_{18} \mathrm{BrNO}_{5}: \mathrm{C}, 56.77 ; \mathrm{H}, 4.08 ; \mathrm{Br}, 17.99 ; \mathrm{N}, 3.15 \%$.

\section{Conclusions}

We believe that the reported method offers a mild, simple, efficient and one-pot synthetic method for the preparation of sterically congested 2,2-disubstituted indane1,3-dione derivatives from Passerini multicomponent reaction of indane-1,2,3-trione. The reaction proceeds smoothly and cleanly under mild conditions and no side reactions were observed. The products were obtained in excellent yields. Their ease of work-up, high yields and mild reaction conditions make it a useful addition to modern synthetic methodologies. Other aspects of this process are under investigation.

\section{Acknowledgments}

The authors are thankful to the Zanjan University for partial support of this work.

\section{References}

1. Zhu, J.; Bienayme, H.; Multicomponent Reactions, Wiley-VCH: Weinheim, 2005.

2. Krasavin, M.; Tsirulnikov, S.; Nikulnikov, M.; Kysil, V.; Ivachtchenko, A.; Tetrahedron Lett. 2008, 49, 5241; Orru, R. V. A.; Greef, M.; Synthesis 2003, 1471; Litvinov, V. P.; Russ. Chem. Rev. 2003, 72, 69;

3. Yavari, I.; Hossaini, Z.; Sabbaghan, M.; Mol. Diversity 2006, 10, 479; Yavari, I.; Sabbaghan, M.; Hossaini, Z.; Mol. Diversity 2007, 11, 1.

4. Shaabani, A.; Soleimani, E.; Sarvary, A.; Rezayan, A. H.; Bioorg. Med. Chem. Lett. 2008, 18, 3968.
5. Banfi, L.; Basso, A.; Cerulli, V.; Guanti, G.; Riva, R.; J. Org. Chem. 2008, 73, 1608.

6. Russowsky, D.; Lopes, F. A.; da Silva, V. S. S.; Canto, K. F. S.; Montes D’Oca, M. G.; Godoi, M. N.; J. Braz. Chem. Soc. 2004, 15, 165; Vieira, Y. W.; Nakamura, J.; Finelli, F. G.; Brocksom, U.; Brocksom, T. J.; J. Braz. Chem. Soc. 2007, 18, 448.

7. Trost, B. M.; Acc. Chem. Res. 2002, 35, 695.

8. Ugi, I.; Werner, B.; Dömling, A.; Molecules 2003, 8, 53; Ugi, I.; Pure Appl. Chem. 2001, 73, 187; Vercillo, O. E.; Andrade, C. K. Z.; Wessjohann, L. A.; Org. Lett. 2008, 10, 205.

9. Ugi, I.; Meyr, R.; Fetzer, U.; Steinbrückner, C.; Angew. Chem. 1959, 71, 386; Ugi, I.; Steinbrückner, C.; Angew. Chem. 1960, $72,267$.

10. Leon, F.; Rivera, D. G.; Wessjohann, L. A.; J. Org. Chem. 2008, 73, 1762 .

11. Banfi, L.; Guanti, G.; Riva, R.; Chem. Commun. 2000, 11, 985.

12. Ugi, I.; Angew. Chem., Int. Ed. 2000, 39, 3168.

13. Hulme, C.; Gore, V.; Curr. Med. Chem. 2003, 10, 51.

14. Banfi, L.; Guanti, G.; Riva, R.; Basso, A.; Calcagno, E.; Tetrahedron Lett. 2002, 43, 4067.

15. Hoffmann, R.; Angew. Chem., Int. Ed. 2001, 40, 3337.

16. Neo, A. G. J.; Delgado, J.; Polo, C.; Marcaccini, S.; Marcos, C. F.; Tetrahedron Lett. 2005, 46, 23.

17. Achatzb, S.; Dömling, A.; Bioorg. Med. Chem. Lett. 2006, 16, 6360.

18. Karimi, A. R.; Rajabi Khorrami, A.; Alimohammadi, Z.; Mohammadi, A. A.; Mohammadizadeh, M. R.; Monatshefte für Chemie 2006, 137, 1079.

19. Passerini, M.; Simone, L.; Gazz. Chim. Ital. 1921, 51,126.

20. Passerini, M.; Gazz. Chim. Ital. 1921, 51,181.

21. Basso, R.; Banfi, L.; Riva, R.; Piaggio, P.; Guanti, G.; Tetrahedron Lett. 2003, 44, 2367.

22. Falck, J. R.; Manna, S.; Tetrahedron Lett. 1981, 22, 619; Schmidt, U.; Weinbrenner, S.; J. Chem. Soc., Chem. Commun. 1994, 1003.

23. Baker, R. H.; Stanonis, D.; J. Am. Chem. Soc. 1951, 73, 699.

24. Souldozi, A.; klepokura, K.; Lis, T.; Ramazani, A.; Z. Naturforsch., B: Chem. Sci. 2007, 62, 835.

25. Souldozi, A.; Ramazani, A.; Tetrahedron Lett. 2007, 48, 1549.

26. Souldozi, A.; Ramazani, A.; Bouslimani, N.; Welter, R.; Tetrahedron Lett. 2007, 48, 2617.

27. Ahmadi, E.; Ramazani, A.; Nekomanesh Haghighi, M.; Tetrahedron Lett. 2007, 48, 6954.

Received: August 9, 2008

Web Release Date: January 15, 2009 